\title{
Simultaneous Opponensplasty and Free Gracilis Muscle Flap for Thenar Reconstruction after Arteriovenous Malformation Resection
}

\author{
Elena Garcia-Vilariño1, Alessandro Thione ${ }^{1} \quad$ Enrique Salmeron-Gonzalez ${ }^{1}$ \\ Alberto Sanchez-Garcia ${ }^{1}$ Alberto Perez-Garcia ${ }^{1}$
} ${ }^{1}$ Department of Plastic Surgery, Hospital Universitario y Politécnico
La Fe, Valencia, Spain

Indian J Plast Surg 2030;53:147-149

\begin{abstract}
Address for correspondence Alessandro Thione, MD, Department of Plastic Surgery, Hospital Universitario y Politécnico La Fe, Avda. Fernando Abril Martorell 106, Valencia, 46026, Spain (e-mail: althione@gmail.com).
\end{abstract}

\begin{abstract}
We report the case of a thenar eminence arteriovenous malformation presenting with

Keywords

- thenar reconstruction

- arteriovenous

malformation

- opponensplasty

- free gracilis muscle

flap continuous growth and pain that was treated with surgical excision after embolization. Extracapsular resection compromised thenar muscles which function was reconstructed with extensor indicis proprius transfer for opposition and abduction, and neurotized free gracilis muscle flap for opposition and adduction, as well as thenar eminence reconstruction.
\end{abstract}

\section{Introduction}

The treatment of arteriovenous malformations (AVM) can be very challenging. Embolectomy or sclerotherapy followed by complete surgical excision and immediate soft tissue reconstruction are considered the gold-standard treatment. ${ }^{1}$ However, the risk of reappearance, bleeding, and alterations of the collateral vascularization are an important limitation, as well as the need for large margins that may oblige to sacrifice contiguous structures. ${ }^{1}$

We report a case of a high-flow AVM on the thenar eminence in which excision endangered the left thumb vascularization and obliged to completely resect the thenar muscles. Thenar muscles are in charge of opposition, abduction, and adduction of the thumb and effective reconstruction is essential for adequate hand functioning. ${ }^{2}$

\section{Case Report}

We present the case of a 33-year-old woman with a pulsatile AVM on the left thenar eminence with years of evolution that had largely grown during her pregnancy ( - Fig. 1). She had given birth 10 months earlier and suffered from pain and loss of thumb prehensile strength since then.
Magnetic resonance imaging (MRI) studies showed a vascular malformation with three different pedicles depending on both the radial and ulnar arteries that completely occupied the thenar eminence. It included both palmar neurovascular pedicles of the thumb, extending proximally from the flexor retinaculum of the wrist to the metacarpophalangeal joint (MCP) and from the radial edge of the first metacarpal bone to the radial edge of the second metacarpal bone. It included flexor pollicis longus (FPL), flexor carpi radialis (FCR), part of the flexor digitorum profundus (FDP) and superficialis (FDS) on their radial side, and displaced flexor pollicis brevis (FPB) and FPL, opponens pollicis (OP), abductor pollicis brevis (APB), and adductor pollicis (AP).

A combined treatment with embolization and surgical resection was offered due to the great pain, functional disorder, and progressive growth. Arterial embolization with coils through both cubital and radial arteries was performed before surgical resection on the same day. The thenar eminence was approached directly, opening the flexor retinaculum to identify FPL, the recurrent motor branch of the median nerve and the collateral branches of the thumb. Extracapsular dissection of the AVM was done and resection included FPB, APB, OP, and AP allowing to preserve FPL in
DOI https://doi.org/ $10.1055 / \mathrm{s}-0040-1709958$ ISSN 0970-0358.
License terms

() (1) $\Theta \circledast$ 


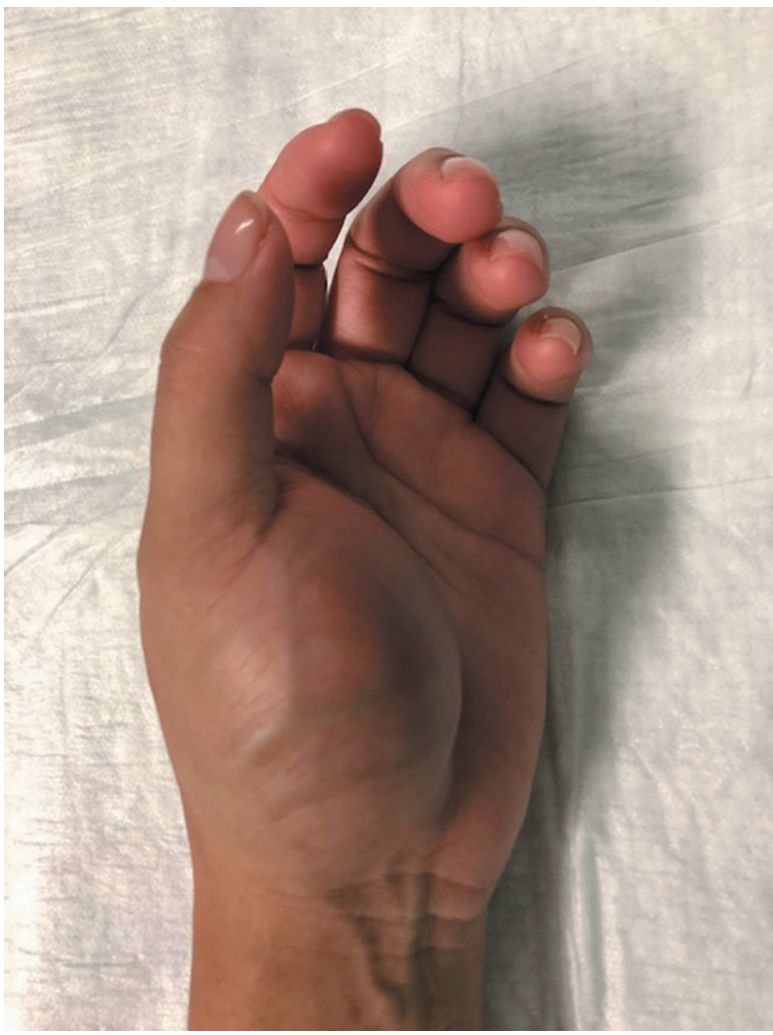

Fig. 1 Preoperative image showing AVM affecting the left thenar eminence. AVM, arteriovenous malformations.

continuity, as well as both collateral nerves of the thumb. The recurrent motor branch of the median nerve was also included in the resection and its distal end was marked with 6-0 nylon suture at the base of the first metacarpal bone (-Fig. 2). Direct closure was performed. Distal capillary refill of the fingers was assessed by the end of the procedure.

Thenar reconstruction was performed 3 weeks later due to logistic reasons. A Burkhalter's opponensplasty and free gracilis muscle flap were performed. Tunneling of extensor indicis proprius (EIP) subcutaneously distal to the pisiform bone was the first step. Distance from the flexor retinaculum to MCP was measured, and contralateral gracilis muscle was harvested full size with the necessary length. Gracilis nerve neurorrhaphy to the recurrent motor branch of the median nerve was performed and free gracilis muscle flap was insetted by anchoring the distal end of the muscle to the distal stump of the APB and OP and the proximal end to the ulnar side of the flexor retinaculum ( - Fig. 3). EIP, deep to the gracilis, was then tightened and anchored to APB and the capsule of the MCP. Finally, the vascular anastomosis was performed; the artery was anastomosed end to side to the radial artery and the veins to superficial veins at the anatomical snuffbox.

Opponensplasty was functional at 1 moth after cast removal and there was full range of movement with gracilis contraction clinically observed and palpable at 3-month postoperatively (-Fig. 4; Video 1).

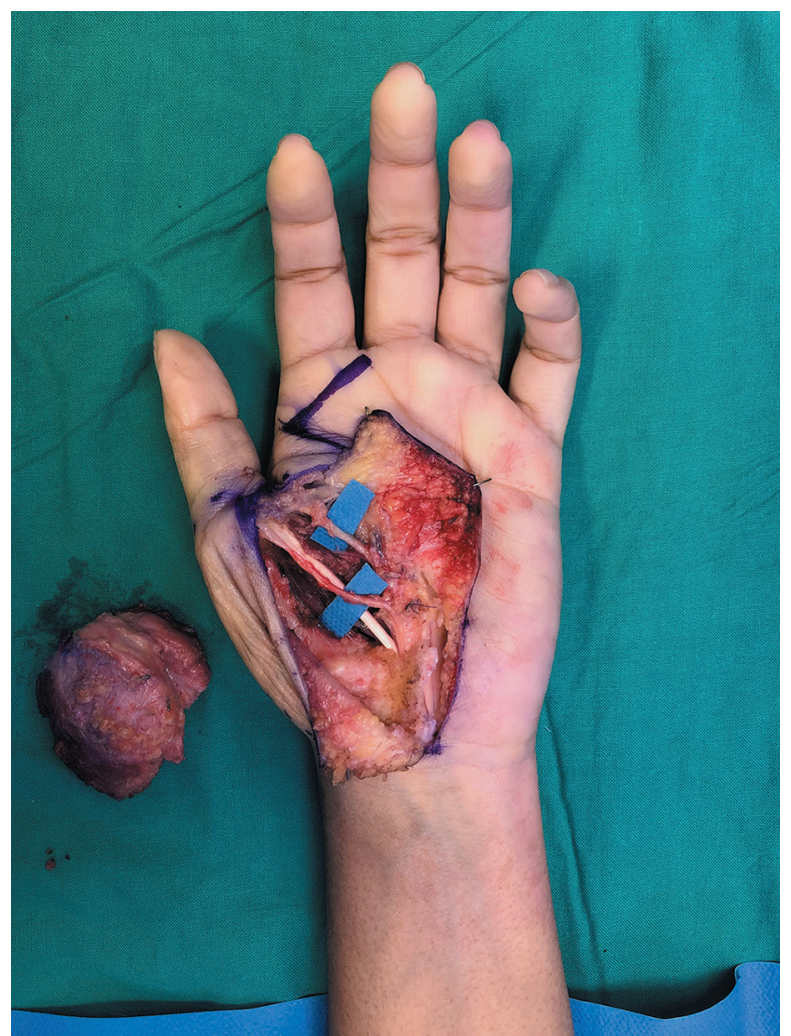

Fig. 2 AVM after resection is shown on the left side of the image. FPL can be seen in continuity, as well as both collateral nerves of the thumb. The distal end of the recurrent motor branch of the median nerve referenced with nylon can also be identified at the base of the first metacarpal bone. AVM, arteriovenous malformations.

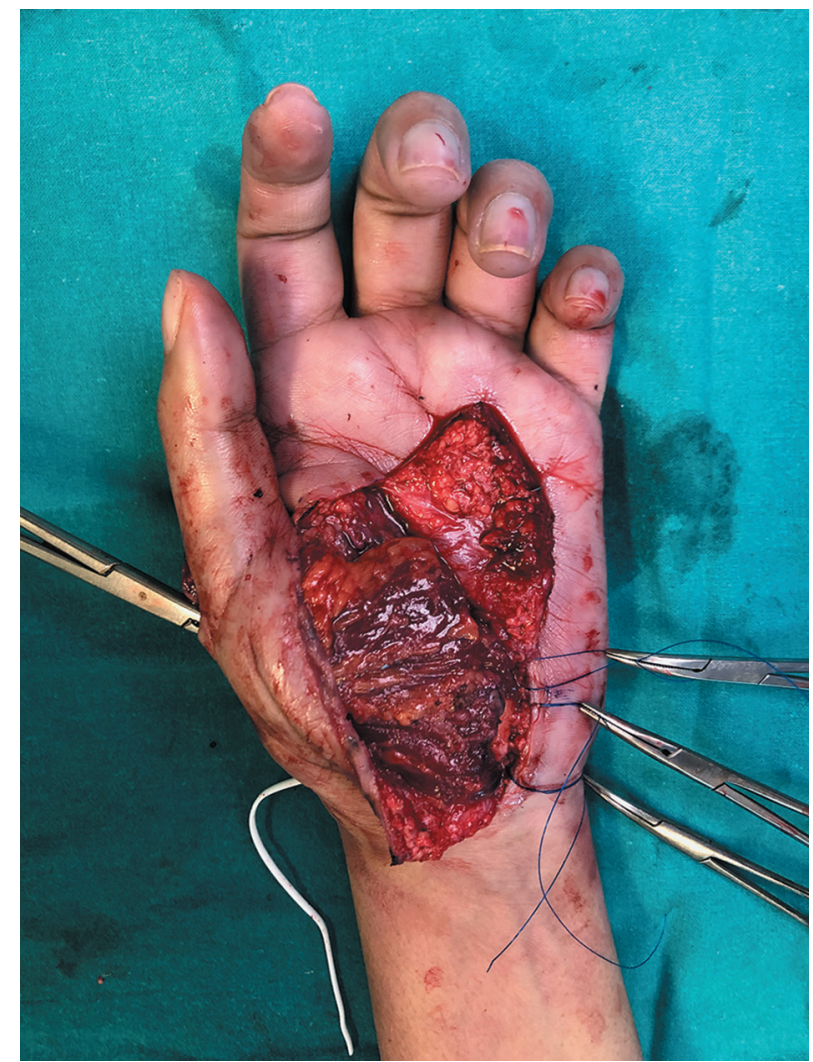

Fig. 3 Intraoperative image showing gracilis muscle flap insetting on the thenar eminence 


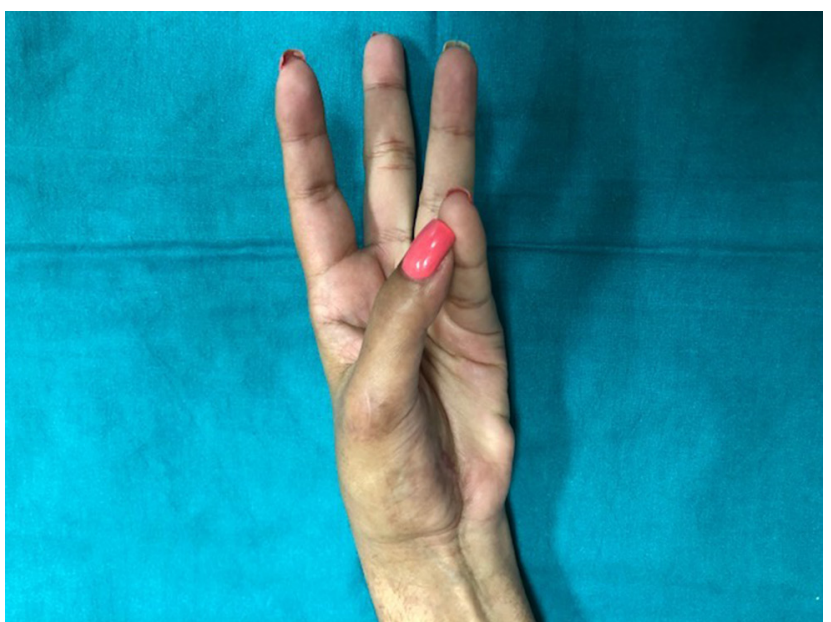

Fig. 4 Postoperative image after 3 months during opposition.

\section{Video 1}

Clinical results are shown in this video. Opposition, abduction, and adduction can be individually observed. Online content including video sequences viewable at: https://www.thieme-connect.com/products/ejournals/ html/10.1055/s-0040-1709958.

\section{Discussion}

Thenar muscles and APB are in charge of thumb opposition and adduction, allowing for precision grip and prehensile tasks. Therefore, muscular resection in this area involves major functional disability. ${ }^{2}$

Usual reconstructive approach includes tendon or muscle transfer, when available, within the hand. Multiple tendon transfers are commonly used for opponensplasty. EIP transfer for opponensplasty was popularized by Burkhalter et al in the 1970s. ${ }^{3}$ Although it has not been the most popular option, this technique has proved good functional outcomes with minimal donor site morbidity and better line of action when compared with other tendon transfers as FDS. ${ }^{4}$

In our case, Burkhalter's opponensplasty was performed with the aim of recovering partial function, while the gracilis flap earned function and strength. However, after 3 months, gracilis muscle function was already visible and both opposition and adduction were more precise. While EIP was mainly placed for opposition and abduction, the muscular flap was placed on the ulnar side allowing not only opposition but also adduction. The use of the recurrent thenar branch to innervate the gracilis muscle would allow a more natural thumb adduction. Moreover, the free muscle transfer provided a suitable bulk for thenar eminence reconstruction, less scar adhesions and a better sliding surface for the tendon transfer. Tendon and muscle transfers within the hand require postoperative reeducation and they do not provide soft tissue, coverage, or reconstruction of lost thenar bulk unlike a free muscle transfer ${ }^{5}$; for this reason, combining both techniques allow in our opinion for a better functional and aesthetic result with a shorter period of rehabilitation.

\section{Conclusion}

The combination of both opponensplasty and free gracilis muscle flap allow a satisfactory result recovering not only thumb opposition and abduction but also adduction of the thumb in less than 3 months, as in our case. Therefore, we consider the combination of both techniques for thenar reconstruction satisfactory functional, aesthetically, and in terms of time until restoration.

\section{Conflict of Interest}

None declared.

\section{References}

1 Ranieri M, Wohlgemuth W, Müller-Wille R, et al. Vascular malformations of upper and lower extremity - from radiological interventional therapy to surgical soft tissue reconstruction an interdisciplinary treatment. Clin Hemorheol Microcirc 2017;67(3-4):355-372

2 Baker PA, Watson SB. Functional gracilis flap in thenar reconstruction. J Plast Reconstr Aesthet Surg 2007;60(7):828-834

3 Burkhalter W, Christensen RC, Brown P. Extensor indicis proprius opponensplasty. J Bone Joint Surg Am 1973; 55(4):725-732

4 Lemonas P, Laing T, Ghorbanian S, Malahias M, Ragoowansi R. Extensor indicis proprius opponensplasty - the Burkhalter revisited. J Hand Microsurg 2012;4(2):47-49

5 Davis TRC, Barton NJ, Median nerve palsy. In: Green DP, Hotchkiss RN, Pederson C, eds. Green's Operative Hand Surgery. 4th ed. Vol. 2. Philadelphia, PA: Churchill Livingstone; 1999:1497-1525 